\title{
Expression of glycogen synthase kinase $3 \beta$ in nephrotic syndrome
}

\author{
Sepideh Zununi Vahed \\ Kidney Research Center \\ Tabriz University of Medical Sciences \\ Tabriz, Iran \\ sepide.zununi@gmail.com
}

\author{
Elham Ahmadian \\ Kidney Research Center \\ Tabriz University of Medical Sciences \\ Tabriz, Iran \\ ahmadian.elham@yahoo.com
}

\author{
Mohammadreza Ardalan \\ Kidney Research Center \\ Tabriz University of Medical Sciences \\ Tabriz, Iran \\ ardalan34@yahoo.com
}

\begin{abstract}
Aberrant expression of glycogen synthase kinase-3 (GSK-3ß) in kidney cells has a harmful role in podocyte injury. In this work, we found that dysregulated levels of GSK$3 \beta$ may be involved in the pathogenesis of nephrotic syndrome, kidney disease with proteinuria, with different etiology.
\end{abstract}

Keywords - Nephrotic syndrome, Proteinuria, Membranous glomerulonephritis, Focal Segmental Glomerulosclerosis, GSK$3 \beta$.

\section{Motivation and Aim}

Glycogen synthase kinase-3 (GSK-3) is a conserved multifunctional serine/threonine kinase that regulates various physiological processes, including gene expression, cell signaling [1], cellular proliferation, apoptosis, and intracellular communication. A detailed understanding of the GSK-3 $\beta$ function and its expression in several pathological conditions will help the clinic manage different kidney diseases

Given the role of GSK-3 $\beta$ in the podocytes injury [2,3], we evaluate its expression levels in PBMCs samples of patients with NS.

\section{Methods}

The expression levels of GSK-3 $\beta$ was evaluated in peripheral blood mononuclear cells (PBMCs) of cases with the most common types of nephrotic syndrome (NS); MGN (membranous glomerulonephritis) and FSGS (focal segmental glomerulosclerosis) using real-time PCR. Sixty cases (30 FSGS and $30 \mathrm{MGN}$ ) were included based on the strict criteria.
The results compared with healthy controls $(n=24)$. The receiver operating characteristic (ROC) curve analysis was used for evaluating the potential of GSK-3 $\beta$ in discriminating cases from controls.

\section{Results}

There were statistically significant increases in GSK-3 $\beta$ expression level in NS $(\mathrm{P}=0.002)$ and FSGS $(\mathrm{P}<0.001)$ groups when compared to controls; however, it was not significant in MGN group $(\mathrm{P}=0.137)$. GSK-3 $\beta$ level was also significantly higher in FSGS group in comparison to MGN group. ROC curve analysis approved a diagnostic power of GSK-3 $\beta$ in discriminating patients from healthy controls (AUC: $0.72, \mathrm{P}=$ 0.002 ) with high sensitivity and specificity.

\section{ACKNOWLEDGMENT}

Supported by the Kidney Research Center at Tabriz University of Medical Sciences (Grant No. 60546 and 62640).

\section{REFERENCES}

[1] Maurer U. et al. (2014) GSK-3 - at the crossroads of cell death and survival. Journal of Cell Science. 127, (Pt 7), 1369-78.

[2] Li C. et al. (2016) The beta isoform of GSK3 mediates podocyte autonomous injury in proteinuric glomerulopathy. The Journal of Pathology. 239, (1), 23-35.

[3] Xu W., Ge Y., Liu, Z. (2014) Glycogen synthase kinase 3beta dictates podocyte motility and focal adhesion turnover by modulating paxillin activity: implications for the protective effect of low-dose lithium in podocytopathy. The American Journal of Pathology. 184, (10), 27422756. 